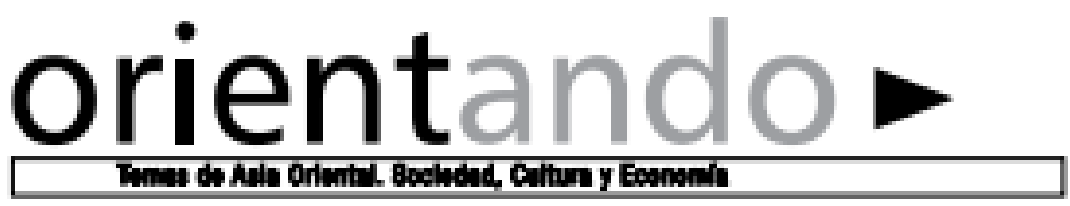

Año 10. Número 21. Octubre 2020-Marzo 2021

ISSN 2007-5723

Centro de Estudios China-Veracruz/Universidad Veracruzana

\title{
Cooperativas rurales de suministro y comercialización en china: modelo de economía solidaria
}

\author{
Cristian Hernández
}

\section{Resumen:}

Las cooperativas rurales de suministro y comercialización en China desarrollaron un modelo exitoso de comercialización mediante la estrategia de e-commerce $\mathrm{O} 2 \mathrm{O}$ (online-offline), que establece vías multicanal para distribuir a mayor escala bienes y servicios, obteniendo un mejor rango de maniobra y negociación ante los intermediarios. Este enfoque coadyuvó a que el sector agropecuario se fortaleciera y aprovechara las oportunidades de la apertura comercial del gigante asiático, esto es, producción rural bajo un esquema de trabajo cooperativo con las ventajas de colocación del libre mercado. El modelo chino otorga una pauta para impulsar al sector cooperativo y fortalecer las prácticas de economía solidaria en Xalapa, considerando los elementos esenciales de la experiencia china y las características de las cooperativas regionales, se configura un dechado que añade valor a la distribución de bienes y servicios en canales eficientes para contrarrestar los problemas de comercialización y se promueva el desarrollo de las cooperativas.

Palabras clave: Cooperativas rurales de suministro y comercialización, China, economía solidaria, 020 (online-offline).

\begin{abstract}
:
Rural supply and marketing cooperatives in China developed a successful marketing model through the online-offline E-commerce strategy, which establishes multichannel routes to distribute goods
\end{abstract}


and services on a larger scale, obtaining a better range of maneuvering and negotiation before intermediaries. This approach helped the agricultural sector strengthen and seize the opportunities for the Asian giant's trade opening, i.e. rural production under a cooperative work scheme with the advantages of free market placement. The Chinese model provides a guideline for boosting the cooperative sector and strengthening solidarity economy practices in Xalapa, taking into account the essential elements of Chinese experience and the characteristics of regional cooperatives, setting up a slap that adds value to the distribution of goods and services in efficient channels to counteract marketing problems, and promotes cooperative development.

Keywords: Rural supply and marketing cooperatives, China, solidarity economy, 020 (onlineoffline).

\section{Introducción}

La práctica cooperativista en México cuenta con buenos referentes como la cooperativa Pascual, Cruz Azul, Tosepan, etc., empresas sociales con proyección internacional y que compiten favorablemente en los diferentes mercados, sin embargo, esto no quiere decir que cada cooperativa que nace esté destinada exactamente al mismo éxito, puesto que hay factores endógenos y exógenos que determinarán el rumbo de la misma. La actividad cooperativa demanda reivindicarse en pensamiento y acción respecto a la motivación particular en armonía con la motivación colectiva y como cualquier organización productiva requerirá de un proceso de desarrollo integral que incorpore una perspectiva empresarial que conviva con la dinámica de mercado, haciendo uso de canales de comercialización y estrategias de distribución para colocar los bienes y servicios ofrecidos.

La cooperativa es una figura asociativa con sentido socio-económico que considera a las actividades económicas como el medio para alcanzar sus objetivos sociales y así generar excedentes (ganancias), las cuales contribuirán al desarrollo de la comunidad de socios con apego a los fondos, distribución y programas de responsabilidad social que la misma organización implementa. Su concepto ha evolucionado con el paso del tiempo según la historia de la propia Ley General de Sociedades Cooperativas (LGSC), que anteriormente Orientando. Temas de Asia Oriental. Sociedad, Cultura y Economía. Revista editada por el Centro de Estudios 
permitía a la cooperativa dedicarse a sólo ciertas actividades designadas y no perseguir fines de lucro, como se sostiene en sus distintas versiones de la Ley en 1927, 1933 y 1938, hasta la promulgación de la última reforma de 1994, hoy en día vigente, dónde las cooperativas pueden dedicarse a cualquier actividad siendo lícita y pueden financiarse incluso con opciones respaldadas por capital de riesgo (Izquierdo, 2019). Esto no se considera para nada una pérdida del sentido central de la especificidad cooperativa, porque como sostiene el autor Javier Andrés Silva Díaz, su carácter autónomo y democrático de la organización permanece como el fundamento distintivo de su filosofía de gestión (como se cita en Izquierdo, 2019).

Lo que sostiene hoy en día la LGSC en su artículo 8, es la importancia de la actividad económica en la ecuación cooperativa, que no necesariamente consiste en una preponderancia hacia al ánimo de lucro, porque el capital es meramente un medio, y no el fin en sí; no es la generación del capital por el capital, sino la creación de beneficios equitativos para resolver las necesidades de los socios que conforman una cooperativa, de manera que el capital y las actividades económicas son la vía para el desarrollo integral para la comunidad de socios. De esta concepción, existe una problemática en que las cooperativas, en una buena proporción, carecen de planes, estrategias y plataformas que logren la comercialización de los bienes y servicios que producen a una mayor escala, promoviendo la capitalización de la sociedad en un corto plazo.

Aunque México posee una gran tradición cooperativista, no ha habido grandes avances debido al paternalismo del Estado que ha impedido el desarrollo de la práctica cooperativista como ha ocurrido con otros países de América Latina (Fritz-Krockow, 1986). La mayoría de los cooperativistas no terminan de concebir al modelo cooperativo siendo compatible con el mercado, dejando de lado la importancia de la comercialización de bienes y servicios, factor determinante para su existencia y éxito. No importa la naturaleza de las actividades que realice la organización, ésta necesitará colocar en el mercado un determinado volumen de ventas para generar ingresos que contribuyan para cubrir sus pasivos, de lo contrario los propietarios abandonarían su participación en la cooperativa debido a la incosteabilidad de las actividades productivas que desarrollan (Rojas, 1984).

Orientando. Temas de Asia Oriental. Sociedad, Cultura y Economía. Revista editada por el Centro de Estudios 
A menudo observamos productos y servicios en el mercado, que quizá no son de tan buena calidad, pero que de alguna u otra forma se adquieren con facilidad a precios accesibles y desde la comodidad de casa con tan solo un par de clicks: ¿a qué se debe esto?, a un diseño estratégico que hace llegar los productos al consumidor de manera oportuna, eficiente y eficaz, es decir, se logró descifrar la en la que el consumidor elige el producto y/o servicio, de esta manera se crea valor en la estructura del negocio.

Las Cooperativas Rurales de Suministro y Comercialización (CRSC) de China responden ante los retos de distribución del sector cooperativo, su rol y estructura son descritos por Guo, Henehan y Schmit (2008) a través de un análisis que aborda los problemas potenciales, los retos relacionados con el desempeño comercial y las estrategias que impulsan el desarrollo de las cooperativas, todo esto, en el contexto del largo proceso de desarrollo histórico y operacional durante las seis fases de la reforma desde 1980 hasta los tiempos actuales. Los resultados obtenidos brindan pautas de enfoque durante la implementación de estrategias similares: la reconstrucción del sector primario, la importancia de la educación cooperativa y la necesidad de comprender su práctica y gestión.

La actividad económica de las cooperativas en la China rural ha tenido un importante desarrollo gracias a la convergencia de dos elementos determinantes: 1) que existe una reserva histórica de valores y principios sociales que brindan idoneidad ante iniciativas de trabajo comunitario, 2) que el gobierno chino implementó el disponer recursos para organizar a las familias en cooperativas rurales de suministro y comercialización, las cuales tienen como base la implementación de un modelo de e-commerce, que propicia un mayor desplazamiento de sus productos, trayendo beneficios importantes al área rural: por ejemplo, los campesinos que se organizan en una cooperativa rural tienen un ingreso entre 20 y $30 \%$ más elevado que el ingreso promedio de los campesinos (Poirier, Hitchman y Theveniaut, 2011).

El modelo de CRSC fue impulsado por el Estado para promover el abasto y la colocación de los productos rurales, toda vez que los productores pudieran asegurar ingresos directos por sus mercancías. Sin embargo, no todo lo que se habla en el sector cooperativo Orientando. Temas de Asia Oriental. Sociedad, Cultura y Economía. Revista editada por el Centro de Estudios 
es, ni siquiera en China, del todo ideal, pues la implementación de estos programas demostró que pese al interés del gobierno en reconstruir a las prácticas económicas comunitarias dedicadas al sector primario, se reconocen los retos de comprensión que el modelo de negocios cooperativo representa tanto para gerentes, granjeros, legisladores y campesinos (Guo, Henehan y Schmit, 2008).

El modelo de CRSC además de considerar el carácter organizativo de la figura cooperativa, ha puesto en el centro asimismo a la comercialización de los bienes y servicios rurales, aprovechando las herramientas de la potencia tecnológica china, mediante el modelo de e-commerce $\mathrm{O} 2 \mathrm{O}$, el cual puede promover el desarrollo regional involucrando a la comunidad local, a través de la integración del servicio local en línea en una estructura a gran escala. El O2O tiene un vasto potencial para integrar al comercio tradicional y en línea (Yue, 2016).

Son muchos los sectores donde el modelo $\mathrm{O} 2 \mathrm{O}$ ha traído importantes experiencias de éxito, y un caso representativo es en el sector de los vegetales orgánicos producidos por campesinos en la provincia de Hubei, donde han aprovechado la creciente popularidad de los productos orgánicos ofreciendo a millones de consumidores una plataforma de productos orgánicos baratos, de calidad, y de fácil y rápido acceso, beneficiando los ingresos de los productores (Guo et al., 2018). Pasquier (2015), afirma que China cuenta con una gran plataforma tecnológica que permite que una gran cantidad de personas estén conectadas a un teléfono móvil, más que en cualquier otra parte del mundo. Por ello China lidera la revolución de ventas $\mathrm{O} 2 \mathrm{O}$, con una estrategia digital que atrae a personas a las tiendas físicas para generar interacción online-to-offline $(\mathrm{O} 2 \mathrm{O})$. China abordó la problemática de las prácticas rurales comunitarias de manera ingeniosa, donde el gobierno no buscó erradicar a los esfuerzos comunitarios, sino brindar apoyo para la configuración e ingreso a las ventajas comerciales de la China urbana.

\subsection{La economía solidaria}

Orientando. Temas de Asia Oriental. Sociedad, Cultura y Economía. Revista editada por el Centro de Estudios China-Veracruz de la Universidad Veracruzana, México (Centro de Estudios APEC) / año 10 / número 21/ 
En América Latina se ha vuelto popular el término "economía social solidaria” (ESS), para referirse a las prácticas socioeconómicas alternativas al sistema de producción capitalista; una especie de concepto híbrido que hace referencia a una combinación de los elementos más sobresalientes de dos conceptos: social y solidaria, esto bajo un enfoque "transformador" respecto a la forma tradicional de generar economía. Asimismo, es común que algunos autores utilicen el concepto de economía solidaria de manera alternativa al de economía social, y viceversa, formas que son aceptadas y se han popularizado principalmente en documentos académicos de las últimas dos décadas (Ávila y Pérez, 2019). Si “social” y "solidaria", no son concretamente sinónimos, existe una vinculación conceptual que es necesario abordar para una mayor comprensión teórica.

Por un lado, la economía social ha adquirido durante las últimas tres décadas una especial importancia en diversos países de Europa y América Latina: países como España, Alemania, Francia, Argentina, Costa Rica, Colombia y demás han difundido casos relevantes para el desarrollo de sus economías preponderantemente supeditadas a modelos de producción que priorizan la generación de capitales sobre el desarrollo integral de las personas que conforman a las organizaciones. La economía social ha manifestado, tanto en el plano teórico como en la práctica, ser un esquema productivo que genera experiencias concretas basadas en la asociatividad con actividades económicas alternativas de base colaborativa (Martínez, 2013). De manera formal, el término economía social aparece en la literatura económica por primera vez en 1830 de la mano del economista liberal francés Charles Dunoyer. Sin embargo, es hasta finales del siglo XIX cuando se perfilan los elementos del concepto moderno inspirado en los valores del asociacionismo democrático, del mutualismo y del cooperativismo (Galindo, 2009).

Debido a que la economía social es una búsqueda teórica y práctica de formas alternativas de hacer economía, basadas en la solidaridad y el trabajo, en la actualidad el concepto engloba una serie muy diversa de experiencias y por consiguiente, es difícil definir la economía social en un concepto único, aunque sí existen conceptualizaciones concretas de autores que identifican sus elementos básicos. Por ejemplo, José Luis Coraggio, economista

Orientando. Temas de Asia Oriental. Sociedad, Cultura y Economía. Revista editada por el Centro de Estudios 
argentino, afirma que este tipo de economía es social porque produce sociedad y no sólo ganancias económicas, porque no está orientada totalmente a las utilidades y la acumulación de capital por el capital. La economía social tiene el poder de cuestionar la delimitación que posee la ciencia económica y de alimentar una reflexión más general sobre las características e instituciones de la economía (Laville y García, 2009). Lo que quiere demostrar es que se puede lograr un desarrollo con equidad, una empleabilidad con justicia, el crecimiento económico sin lucro e inclusión social con perspectivas democráticas. En otras palabras, puede definirse como un movimiento socioeconómico que se fundamenta en un esquema de valores y principios orientados a la construcción de una economía centrada en las personas, su desarrollo integral y el fomento de prácticas de cooperación y solidaridad en sus comunidades (Oulhaj y Saucedo, 2013).

La economía solidaria, por su parte, nace a partir de los años 80 del siglo XX en Francia, con la voluntad explícita de cambio social; partiendo de una lógica donde, ni las acciones del sector público ni las del sector privado, han logrado resolver las necesidades sociales modernas y los riesgos de exclusión, que parten de las relaciones sociales que generan diversas formas de producción, distribución y consumo integradas en una dinámica horizontal y vertical, la economía solidaria corresponde a una unidad asociativa con carácter empresarial que produce bienes y servicios como resultado de la libre asociación de personas físicas que se integran para desarrollar eficientemente bienes y/o servicios para sus propias necesidades o las de la comunidad (Arango, 2005).

El componente que configura el perfil de la economía solidaria es la correspondencia al mercado, la cual se da a través de la interacción de tres polos principales: el mercado, el estado y la reciprocidad. Estos polos se corresponden entre sí con los principios de mercado, de redistribución y de reciprocidad. Ésta última descansa sobre el asociacionismo donde no todo recae en un intercambio monetario, sino en el de la sociabilidad primaria y con una dinámica de intercambio no monetario. En otras palabras, aunque con la palabra "mercado" podríamos entender el carácter mercantil y/o monetario de la economía solidaria, no quiere decir que por ello puede reducirse estrictamente a esos términos, porque el enfoque de la

Orientando. Temas de Asia Oriental. Sociedad, Cultura y Economía. Revista editada por el Centro de Estudios 
Economía Solidaria brinda una articulación inédita entre los tres polos del sistema, a través de experiencias concretas híbridas de economías de mercado (privadas), de no mercado (públicas) y no monetarias (voluntariados).

Pese a que existe un concepto acuñado, es interesante cómo la concepción puede cambiar según las regiones: para América Latina, la economía solidaria es una fuerza de transformación social portadora de un proyecto de sociedad alternativa a la mundialización neoliberal, un mero proyecto global alternativo al capitalismo. Para Europa, la economía solidaria es compatible con el mercado y con el Estado. Dicho de otra forma, una empresa de la economía solidaria es una organización dedicada a actividades industriales, mercantiles o de prestación de servicios que busca tener un impacto social o ambiental positivo y medible.

Dicho impacto debe ser de forma autosustentable, es decir, que genere sus propios recursos para seguir desarrollando sus actividades (Galindo, 2009). La economía solidaria se conceptualiza como una economía alternativa frente a la incapacidad estructural del sistema capitalista para propiciar bienestar a todos aquellos que viven de su trabajo y se halla dentro de la denominada economía social, que encuentra sus bases en la convivencia fraternal del ser humano en lo político y lo social y la cooperación en lo económico para que todos se beneficien. No tiene como base fundamental la producción sin fines de lucro, sino que esta economía busca el bienestar de todos (Olmedo, 2017). La economía solidaria es una práctica que nace del tronco común de la economía social, siendo una perspectiva que replantea las relaciones económicas desde parámetros diferentes, considerando la lógica del capital, la mercantilización creciente de las esferas públicas y privadas y la búsqueda de máximo beneficio, asimismo, persigue el construir relaciones de producción, distribución, consumo y financiación basadas en la justicia, la cooperación, la reciprocidad y la ayuda mutua de frente a la acumulación del capital poniendo a las personas y su trabajo en el centro del sistema económico, otorgando a los mercados un papel instrumental siempre al servicio del bienestar de todas las personas y de la reproducción de la vida en el planeta (Pérez, Etxezarreta y Guridi, 2008).

\subsection{Cooperativas: un modelo de economía solidaria}

Orientando. Temas de Asia Oriental. Sociedad, Cultura y Economía. Revista editada por el Centro de Estudios China-Veracruz de la Universidad Veracruzana, México (Centro de Estudios APEC) / año 10 / número 21/ 
En el andamiaje legal, se identifica a la Economía Social Solidaria dentro del sector social de la economía, contemplado como un factor responsable de contribuir al desarrollo económico del país desde la Constitución Política de los Estados Unidos Mexicanos (CPEUM) en el art. 25 párrafo IV, donde no sólo enuncia al sector público y privado, sino que también al sector social, y "sin menoscabo de otras formas de actividad económica" éste debe contribuir al desarrollo económico de la nación. En el mismo artículo, pero en el párrafo VIII, se menciona explícitamente a las cooperativas como una forma de organización socioproductiva, encomendando a la ley el establecer mecanismos para facilitar la organización de la actividad económica del sector social.

Aunque existen numerosas expresiones de la economía social y solidaria, se puede decir que las cooperativas son el brazo operativo más regulado y representativo, que se sostiene histórica y fehacientemente al demostrar sus oportunidades de organización social para el trabajo en el mundo; en países como Alemania, Inglaterra, Suecia, Dinamarca, Israel, etc., el sector cooperativo ha alcanzado niveles de desarrollo altos, gracias, entre otras cosas, al impulso que sus gobiernos han brindado a las cooperativas establecimiento condiciones que promueven su desarrollo, integración y relevancia económica en sus sociedades. La sociedad cooperativa es, básicamente, la expresión jurídica de la práctica cooperativista. México cuenta con un marco legal interesante, aunque no suficiente, que ha motivado su avance.

Las cooperativas comienzan a tener un lugar a partir de la inserción de un capítulo especial sobre las Sociedades Cooperativas en el Código de Comercio de 1889. Casi cuatro décadas después, en 1927, el presidente Plutarco Elías Calles formuló un proyecto de ley de cooperativas, que fue aprobado y publicado por el Congreso de la Unión, inspirado por el sistema de organización cooperativista europeo, que conoció durante un viaje por ese continente. Sin embargo, es hasta el 15 de febrero de 1938, que se publica en el diario oficial la Ley General de Sociedades Cooperativas, durante el gobierno presidencial de Lázaro Cárdenas (García y Vázquez, 2013).

Orientando. Temas de Asia Oriental. Sociedad, Cultura y Economía. Revista editada por el Centro de Estudios China-Veracruz de la Universidad Veracruzana, México (Centro de Estudios APEC) / año 10 / número 21/ 
El sector cooperativo continuó rigiéndose por las mismas disposiciones legales durante cincuenta y seis años, hasta en 1994, durante la gestión del entonces presidente de la República, Carlos Salinas de Gortari, se realizó la reforma a la Ley General de Sociedades Cooperativas (LGSC). Años más tarde, en agosto del 2009, se publica la Ley para Regular las Actividades de las Sociedades Cooperativas de Ahorro y Préstamo, mejor conocida como la LRASCAP, la cual delimitó la regulación de las actividades del brazo financiero de las cooperativas. La promulgación de esta ley no sólo atendió aspectos importantes de la operatividad de las cooperativas de ahorro y préstamo, sino que también propició su desarrollo, de tal forma que al día de hoy es el tipo de cooperativa mejor regulada, organizada y estructurada en México, a diferencia de las otras dos clases contempladas por la LGSC en su artículo 21: las de consumidores, y las de productores de bienes y/o servicios. Estas dos categorías debidamente tipificadas no contemplan una ley especial para regular sus actividades, pese a que son las que registran una mayor población y a la vez suelen ser las más vulnerables, con situaciones más precarias desde su nacimiento y las que se constituyen con mayor frecuencia. Desde la publicación de la LGSC en 1994, no se han hecho reformas significativas a dicha Ley, aunque sí modificaciones sustanciales en la redacción, que no corresponden propiamente a las demandas del sector y que están lejos de considerar las experiencias y recomendaciones de la comunidad cooperativista mexicana.

La LGSC en su artículo 2, define a la cooperativa como una forma de organización social integrada por personas con intereses comunes, basados en los principios de solidaridad, esfuerzo y ayuda mutua, que tienen como propósito el satisfacer necesidades individuales y colectivas, a través de la realización de actividades económicas de producción, distribución y consumo de bienes y servicios. En otras palabras, la cooperativa es una organización centrada en las personas, libremente asociada y autónoma de individuos que se han unido voluntariamente para hacer frente a sus necesidades y aspiraciones económicas, sociales y culturales comunes por medio de una empresa de propiedad conjunta y democráticamente controlada (ICA, 2018). Como organización social que se da entre personas con intereses comunes, éstas se sujetan a principios rectores como la solidaridad, el esfuerzo propio y la

Orientando. Temas de Asia Oriental. Sociedad, Cultura y Economía. Revista editada por el Centro de Estudios China-Veracruz de la Universidad Veracruzana, México (Centro de Estudios APEC) / año 10 / número 21/ 
ayuda mutua para satisfacer las necesidades individuales o colectivas de sus integrantes a través de actividades económicas de producción, distribución, consumo y ahorro y préstamo.

El espíritu cooperativista radica en el servicio al socio, a la comunidad, al sentido de atender las necesidades de sus propios integrantes, quienes democráticamente, determinan el rumbo de su organización. Las cooperativas en el mundo han evolucionado de ser tan sólo un modelo de conquista de mejora de las condiciones de un grupo de trabajadores a ser un motor de desarrollo económico y social para las naciones (Rojas, 2013). El elemento sine qua non que brinda identidad y diferenciación a la figura de la cooperativa es sin duda el código de principios: aquellos lineamientos que darán rumbo objetivo en la aplicación diaria de su ideología y que no son solo concepciones morales en el subconsciente o palabras en un cuadro adornando la recepción de las oficinas de la organización, sino que en realidad son disposiciones legales contenidas en el artículo $6^{\circ}$ de la LGSC, el conjunto de principios deberá ser observado en el actuar cooperativa para el buen funcionamiento y consisten en los siguientes: 1) libertad de asociación y retiro voluntario de los socios; 2) administración democrática; 3 ) limitación de intereses a algunas aportaciones de los socios, si así se pactara; 4) distribución de los rendimientos en proporción a la participación de los socios; 5) fomento de la educación cooperativa y economía solidaria; 6) participación en la integración cooperativa; 7) respeto al derecho individual de los socios de pertenecer a cualquier partido político o asociación religiosa, y 8) promoción de la cultura ecológica.

\subsection{Las cooperativas rurales de suministro y comercialización en China (CRSC)}

La actividad cooperativa en China data de una tradición tan milenaria como su civilización misma, con un extenso reportorio de experiencias exitosas de producción comunitaria, sin embargo, para efectos de esta investigación, y que aunque el sector de las cooperativas agrícolas y rurales en China es muy vasto, con la existencia de las cooperativas de crédito rural, las organizaciones cooperativas comunitarias y las nuevas cooperativas especializadas de agricultores, abordaremos solo el caso de las Cooperativas Rurales de Suministro y Comercialización en China (CRSC). El cual es un modelo de cooperativas impulsado por el Estado implementado para promover el abasto y la colocación de los productos rurales, toda

Orientando. Temas de Asia Oriental. Sociedad, Cultura y Economía. Revista editada por el Centro de Estudios 
vez que los productores aseguren ingresos directos por sus mercancías, asimismo son consideradas como un componente importante del sistema de economía planificada y como una entidad económica por sí sola (Guo et al., 2008).

El rol de las CRSC en China principalmente consiste en: 1) servir a los productores agrícolas y expandir el negocio agrícola; 2) hacer mayor énfasis en el concepto “cooperativa", para dar carácter y fortalecerlo y no solo ser cooperativas de nombre; 3 ) construir una red de servicios comerciales y públicos que cultiven y fortalezcan sus alianzas; 4) promover que las CRSC sean de la propiedad de los mismos productores y sean controladas por ellos mismos; 5) promover el intercambio de bienes y servicios entre las ciudades y las comunidades rurales; 6) asegurar suministros de mercado adecuados para sus actividades de producción; 7) acelerar la gestión industrializada de la agricultura, y 8) proporcionar servicios a los agricultores y las actividades de producción agrícola (Guo et al., 2008).

La estrategia conjunta entre el gobierno y los productores chinos consistió en conformar cooperativas enfocadas en la comercialización, para que tuvieran un mayor margen de maniobra en negociaciones con intermediarios; mejor conocidas como cooperativas rurales de suministro y comercialización (CRSC), llamadas también en inglés como Rural Supply and Marketing Cooperatives (SMCs). Este tipo de cooperativa considera funciones amplias mercadológicamente hablando, porque se enfocan completamente en establecer estrategias multicanales de comercialización para la obtención de suministros y la colocación de los bienes y servicios que producen. Tan solo en 2016, las ventas que lograron colocar ascendieron a 4.78 billones de yuanes, con un aumento de $10.9 \%$ anual (Xinhuanet, 2018). Este histórico desarrollo de las cooperativas rurales de suministro y comercialización se debe principalmente al crecimiento del comercio electrónico y al establecimiento de una plataforma de e-commerce mediante una estrategia multicanal $\mathrm{O} 2 \mathrm{O}$ (online-offline), que el mismo gobierno chino implementó de la mano de la Federación de Cooperativas de Suministro y Marketing de China (All China Federation of Supply and Marketing Cooperatives).

Orientando. Temas de Asia Oriental. Sociedad, Cultura y Economía. Revista editada por el Centro de Estudios China-Veracruz de la Universidad Veracruzana, México (Centro de Estudios APEC) / año 10 / número 21/ 
La estrategia O2O ayuda a las compañías a atraer a más clientes a sus tiendas físicas, mientras ofrecen una experiencia por internet que se complementa con una interacción física, maximizando el beneficio y creando una sinergia para sus productos y servicios offline. Cuando el cliente visita algún establecimiento, la empresa tiene la oportunidad de venderle otros productos al cliente y así ofrecerle la experiencia de un establecimiento físico, los clientes pueden obtener descuentos o beneficios al ordenar en internet y disfrutar de manera presencial durante su visita (Yue, 2016).

La estrategia multicanal $\mathrm{O} 2 \mathrm{O}$ consiste en un proceso de: 1) crear una tienda virtual para cada tienda offline: una representación exacta digital de una tienda física, y los clientes se registran con la posibilidad de hacer pedidos y recoger la mercancía en alguna tienda; 2) establecer un sistema de crédito: con opciones flexibles de pago, transacciones seguras y gestión de quejas; 3) desarrollar transacciones globales: oportunidad de generar comercio con el mundo entero, facilitando el acceso en otros idiomas, servicios de envíos a todo el mundo, socios comerciales, etc. (Fang, 2018). El O2O intenta alcanzar el desarrollo regional envolviéndose con la comunidad local, la cual integrará el servicio online en una estructura de escala larga con un vasto potencial para integrar la economía online-offline (Yue, 2016).

El modelo chino de CRSC consiste en fortalecer la identidad cooperativa, asegurar los suministros para la industrialización de la producción solidaria y promover la comercialización de los bienes y servicios del sector agropecuario, aprovechando las oportunidades de la apertura comercial de China y el gigantesco volumen de su comercio electrónico, esto es: la producción rural de las cooperativas se comercializa con las ventajas del libre mercado a través de las redes económicas solidarias y no solidarias. La configuración de los elementos del modelo permite generar valor en los productos y servicios partiendo de la interacción de la empresa con el consumidor y otros factores, de esta forma, los clientes pagarían conforme a la utilidad, solución y experiencia que se les ofrezca, y no conforme a los costos de producción de la organización, buscando siempre lo de menor precio (Mutis y Ricart, 2008).

Orientando. Temas de Asia Oriental. Sociedad, Cultura y Economía. Revista editada por el Centro de Estudios China-Veracruz de la Universidad Veracruzana, México (Centro de Estudios APEC) / año 10 / número 21/ 
El objetivo de la presente investigación fue implementar el modelo chino de Cooperativas Rurales de Suministro y Comercialización (CRSC) y determinar si los elementos que lo configuran son capaces de impulsar la comercialización de los bienes y servicios de cooperativas en la región Xalapa. Lo anterior mediante una configuración que consideró los factores endógenos y exógenos de estas empresas sociales, para dar viabilidad a la implementación del modelo considerando la estrategia multicanal $\mathrm{O} 2 \mathrm{O}$ (online to offline) en una muestra piloto de cinco cooperativas durante un periodo de un mes (octubre 2020).

\section{Métodos}

La ubicación espacial del presente estudio se desarrolló en cooperativas localizadas en la ciudad de Xalapa, Veracruz, con el aval de la Subdirección de Innovación y Economía Solidaria, perteneciente a la Dirección de Desarrollo Económico del H. Ayuntamiento de Xalapa. En un primer momento, se realizó la aplicación de un diagnóstico a 23 cooperativas desarrollado del 21 al 25 de febrero del 2020, donde se evaluaron las condiciones en ese momento, para determinar cuáles cooperativas serían las más idóneas para aplicar el modelo de Cooperativas Rurales de Suministro y Comercialización (CRSC).

Posteriormente, durante el 26 de agosto se sostuvo una reunión informativa con las cooperativas para darles a conocer los alcances de la investigación y solicitar su participación en la realización del estudio, donde se determinaron los candidatos más idóneos para la aplicación del modelo chino de cooperativas obteniéndose el consenso de los participantes. El modelo anteriormente referido se aplicó durante el periodo del 1 al 31 del mes de octubre de 2020, mes en el cual la semaforización permitió a las cooperativas operar de una manera más completa, derivado de las acciones implementadas para frenar la propagación de la Covid-19 en la ciudad. Se eligió este mes con el propósito de que las condiciones fueran lo más parecidas a las transacciones cotidianas, contemplando el impacto de la pandemia y la contracción del nivel de operaciones ocasionado por las medidas sanitarias de combate ante el mortal virus.

Orientando. Temas de Asia Oriental. Sociedad, Cultura y Economía. Revista editada por el Centro de Estudios China-Veracruz de la Universidad Veracruzana, México (Centro de Estudios APEC) / año 10 / número 21/ 
Durante la aplicación del modelo se realizaron cuatro sesiones de asistencia para resolver los asuntos relacionados con la implementación y monitorear la aplicación y disposición de los recursos materiales, tecnológicos y humanos requeridos durante el desarrollo. Para incrementar la viabilidad en su aplicación se realizaron algunas adaptaciones al modelo original de cooperativas implementado por el gobierno chino.

Como punto de partida, las cooperativas que participaron en este estudio cuentan con características diferentes al modelo original, principalmente en tres aspectos: 1) la génesis organizacional: la función de "suministro y comercialización" no fue la vocación original que dio origen a la cooperativa, sino que fue el consenso en sus necesidades y objetos sociales siendo conscientes de que es necesario desarrollar con mayor énfasis las funciones de comercialización y distribución; 2) la participación operativa: los socios contribuyen directamente en las tareas operativas para generar los bienes y servicios que se ofrecen, es decir, no están dedicados exclusivamente a las tareas de suministro y comercialización y por ende éstas actividades deben delegarse a otros miembros de la sociedad; y 3) la infraestructura disponible: la localización de los establecimientos cuentan con características tecnológicas básicas, pero suficientes para implementar acciones del modelo de CRSC y el $\mathrm{O} 2 \mathrm{O}$.

En cuanto a las similitudes encontramos también tres aspectos: 1) interés público: en China el gobierno dispuso fondos especiales para apoyar la organización en cooperativas (Poirier, 2011), en Xalapa, se reformó la estructura orgánica del H. Ayuntamiento (Gobierno del Estado de Veracruz, 2018), para crear un área específica en la dependencia para impulsar la misma causa; 2) potencial para el desarrollo: tanto en China como en México, las cooperativas son vistas como un instrumento que articula esfuerzos para combatir la pobreza (Xinhuanet, 2019), que resiste a las crisis de manera superior a las empresas capitalistas convencionales (Martínez, 2015) y que suele ser considerada en los planes y proyectos económicos de los gobiernos (Fritz-Krockow, 1986), y 3) ventas de productos locales: las CRSC en China tienen como objetivo impulsar la comercialización de los productos locales

Orientando. Temas de Asia Oriental. Sociedad, Cultura y Economía. Revista editada por el Centro de Estudios China-Veracruz de la Universidad Veracruzana, México (Centro de Estudios APEC) / año 10 / número 21/ 
y la expansión de la red comercial, a través de circuitos de consumo, suministro y distribución que involucren los bienes y servicios producidos por las cooperativas (Xinhuanet, 2019).

Si bien los acontecimientos de un determinado modelo dependen de todo un ecosistema económico, social y tecnológico, los aspectos anteriormente descritos dan la pauta para encontrar los elementos del modelo de CRSC, las diferencias contextuales y los puntos de convergencia, con el propósito de determinar las adaptaciones necesarias para la aplicación del modelo de manera local. Para una mayor comprensión, en la Tabla 1 se presentan los diferentes elementos descritos por Guo et al. (2018) y Fang (2018), y las adaptaciones descritas mencionadas anteriormente.

Tabla 1. Adaptación del modelo de Cooperativas Rurales de Suministro y Comercialización a las cooperativas de la ciudad de Xalapa, Ver.

\begin{tabular}{|l|l|l|l|}
\hline No. & Elemento & Viabilidad & Adaptaciones/Consideraciones \\
\hline \multicolumn{2}{|l|}{ Elementos básicos de las CRSC } & \\
\hline 1 & $\begin{array}{l}\text { Vocación, formación y } \\
\text { congruencia en la propiedad } \\
\text { desempeño de la práctica } \\
\text { cooperativista. }\end{array}$ & $\begin{array}{l}\text { Completa } \\
\text { Se eligieron 5 cooperativas con } \\
\text { madurez suficiente en la gestión } \\
\text { interna, buen desempeño y } \\
\text { operaciones consistentes. }\end{array}$ \\
\hline 2 & $\begin{array}{l}\text { Control, y alianza de los } \\
\text { productores. }\end{array}$ & $\begin{array}{l}\text { Completa } \\
\text { socios es satisfactorio para la } \\
\text { toma de decisiones. }\end{array}$ \\
\hline 3 & $\begin{array}{l}\text { Intercambio entre zonas rural- } \\
\text { urbana y } \\
\text { (suministro yiceversa } \\
\text { comercialización) }\end{array}$ & $\begin{array}{l}\text { Completa } \\
\text { distribuyen/adquieren productos } \\
\text { en la zona de Xalapa, tanto área } \\
\text { rural como urbana. }\end{array}$ \\
\hline
\end{tabular}

Orientando. Temas de Asia Oriental. Sociedad, Cultura y Economía. Revista editada por el Centro de Estudios China-Veracruz de la Universidad Veracruzana, México (Centro de Estudios APEC) / año 10 / número 21/ 


\begin{tabular}{|c|c|c|c|}
\hline 4 & $\begin{array}{l}\text { Apertura } \quad \text { a la } \\
\text { industrialización, asistencia y } \\
\text { servicios a la producción } \\
\text { agrícola }\end{array}$ & Completa & $\begin{array}{l}\text { Las cooperativas participantes } \\
\text { están abiertas a la innovación y } \\
\text { mejoramiento de sus procesos. }\end{array}$ \\
\hline \multicolumn{4}{|c|}{ Estrategia 020 (online-offline) } \\
\hline 1 & $\begin{array}{l}\text { Creación de una tienda virtual } \\
\text { (on-line) por cada tienda } \\
\text { física (off-line). }\end{array}$ & Mediana & $\begin{array}{l}\text { Las cooperativas participantes no } \\
\text { cuentan con una tienda propia } \\
\text { virtuales, pero cuentan con } \\
\text { presencia satisfactoria en medios } \\
\text { digitales para desarrollar } \\
\text { interacciones online-offline. }\end{array}$ \\
\hline 2 & $\begin{array}{l}\text { Sistema de crédito: pagos } \\
\text { flexibles, } \\
\text { seguras y gestión de quejas. }\end{array}$ & Mediana & $\begin{array}{l}\text { Las cooperativas participantes } \\
\text { cuentan con opciones de pago } \\
\text { suficientes para transacciones } \\
\text { electrónicas }\end{array}$ \\
\hline 3 & $\begin{array}{l}\text { Transacciones globales: } \\
\text { comercio con el mundo } \\
\text { entero, envíos globales. }\end{array}$ & Mediana & $\begin{array}{l}\text { Las cooperativas no cuentan con } \\
\text { áreas especializadas para atender } \\
\text { ventas con el exterior, pero } \\
\text { recientemente recibieron } \\
\text { capacitación básica para la } \\
\text { internacionalización, mediante } \\
\text { opciones de e-commerce, } \\
\text { mostrando completa solicitud a lo } \\
\text { que de ahí emane. }\end{array}$ \\
\hline
\end{tabular}

Fuente: Elaboración propia con información de Guo et al. (2018) y Fang (2018).

Orientando. Temas de Asia Oriental. Sociedad, Cultura y Economía. Revista editada por el Centro de Estudios China-Veracruz de la Universidad Veracruzana, México (Centro de Estudios APEC) / año 10 / número 21/ 
De acuerdo al Directorio Estadístico Nacional de Unidades Económicos (DENUE) del INEGI, en 2020 se cuenta con un registro en Xalapa de 33 cooperativas, de las cuales 27 corresponden al sector de autotransporte, 1 de servicios de grúas y 5 al sector escolar. No se tiene estadística registrada de cooperativas en algún otro sector. Sin embargo, con base en datos de la oficina de Desarrollo Económico Municipal del H. Ayuntamiento de Xalapa, se considera que existen a la fecha un total 50 cooperativas registradas en el Padrón Municipal de Cooperativas, las cuales ofrecen productos y servicios de sectores que podrían ser distribuidos a través del modelo de CRSC.

Se consideró conveniente determinar un muestreo de tipo no probabilístico, para los resultados obtenidos en cinco cooperativas que cuenten con al menos las siguientes características: estar debidamente constituidas; con domicilio en la ciudad de Xalapa, Ver.; tener disponibles productos y/o servicios con potencial para ser distribuidos a través de plataformas online; tener al menos un año de operaciones; contar con la infraestructura tecnológica y de personal para responder a la implementación del modelo CRSC, y brindar su consentimiento para participar en la investigación. La elección de las cooperativas candidatas a participar en el estudio se debe al cumplimiento de las características anteriormente enlistadas y su accesibilidad para concurrir debidamente con las acciones y objetivos del presente estudio.

De manera preliminar al instrumento de captación de datos se aplicó, en un primer momento, un diagnóstico a 23 cooperativas de una población total de 50. Estos resultados fueron evaluados para determinar los mejores candidatos para la aplicación del modelo y su futura evaluación del desempeño en las actividades de la cooperativa, lo que brindó un panorama de la accesibilidad, voluntad y viabilidad de la aplicación del modelo.

Posteriormente, al cabo de ocho meses, se implementa el modelo chino de CRSC en las empresas mejor valuadas para su aplicación, por un periodo de 31 días, aplicando el instrumento de captación de datos, recabando información correspondiente a los siguientes aspectos de la cooperativa: aspectos generales; recursos humanos, tecnológicos y técnicos; aprovechamiento de medios digitales para la comercialización; impacto en el nivel de Orientando. Temas de Asia Oriental. Sociedad, Cultura y Economía. Revista editada por el Centro de Estudios 
transacciones e ingresos; configuración de la estrategia de comercialización, y retos enfrentados.

Una vez finalizado el periodo de implementación del modelo, el día 2 de noviembre del 2020 se aplicó el instrumento para recabar los datos correspondientes y proceder al procesamiento, análisis e interpretación de los resultados obtenidos durante la aplicación del modelo de CRSC. Mediante la comparativa de estos parámetros se determinó la viabilidad del modelo para su implementación en el contexto de las cooperativas regionales y la posible solución a los problemas de comercialización que éstas presentan. Aunque los resultados de la aplicación de ambos instrumentos pueden corresponder a dos momentos circunstancialmente distintos: el primero, un escenario "pre-pandemia", y el segundo, un escenario "in-pandemia" pudiendo sesgar los resultados, es precisamente esto lo que representa una oportunidad para que los resultados tengan mayor relevancia y se cumplan los objetivos del presente estudio.

\section{Resultados}

Como se mencionó anteriormente, uno de los propósitos del modelo $\mathrm{O} 2 \mathrm{O}$ (online to offline), es atraer clientes que complementen su experiencia con la organización mediante una visita física al establecimiento utilizando atractivas promociones otorgadas por la vía digital. Sobre la semaforización de la Covid-19 en el estado de Veracruz, durante el mes de octubre se presentaron los estatus color naranja (riesgo alto) y amarillo (riesgo medio), con lo cual las autoridades municipales en materia de comercio otorgaron flexibilidad para los negocios en la ciudad, siempre y cuando se cumpliera con un protocolo sanitario para ingresar a los establecimientos. Las cooperativas no estuvieron exentas y en congruencia a su responsabilidad social, el $100 \%$ de las participantes del estudio contaron con las medidas básicas como el uso obligatorio de cubrebocas para el ingreso, toma de temperatura, la sanitización periódica de espacios y el uso oportuno de gel antibacterial; un rango del 20\%$40 \%$ implementaron otras medidas complementarias como el tapete sanitizante, limitación

Orientando. Temas de Asia Oriental. Sociedad, Cultura y Economía. Revista editada por el Centro de Estudios 
de ingreso, sana distancia, etc. La difusión de la implementación de las medidas sanitarias pertinentes para disminuir las probabilidades de contagio cuidando a los socios, colaboradores y clientes, brindó confianza en su público, de manera que el modelo fue viable pese a la contingencia ocasionada por la Covid-19.

Del total de cooperativas donde se aplicó el modelo, el $80 \%$ ofreció producto gratis y el $60 \%$ precios y presentaciones exclusivas en sucursales; mientras que el $40 \%$, descuentos solo válidos en establecimiento. Estas dos primeras opciones, fueron más eficaces que algunas otras promociones como cupones, amenidades y obsequios de patrocinadores. Considerando el complejo contexto donde se aplicó, tiene sentido encontrar que los consumidores buscaran descuentos extra en los productos que acostumbraban a consumir, incluso si para obtenerlos debían de acudir a algún establecimiento. Hay que resaltar que las estrategias tuvieron éxito para atraer clientes de manera física ya que el $60 \%$ de las cooperativas afirmaron que las iniciativas de promoción en establecimiento funcionaron para que los clientes que habían generado contacto en línea concretaran una visita al establecimiento. Esto no solo trajo beneficio en el sentido de cumplir con el propósito del modelo, sino que también influyó positivamente en las ventas, ya que el $40 \%$ de las cooperativas afirma que en promedio de 5 a 7 de cada 10 personas que visitaron el establecimiento adquirieron algún producto o servicio extra al que inicialmente habían planeado adquirir.

Como se observa en la Figura 1, en febrero del 2020 el 60\% de las cooperativas lograban menos de 100 transacciones mensuales y el 40\% restante un rango entre 100 y 199, ninguna cooperativa lograba superar las 200 transacciones por ventas. Durante el mes de la aplicación del modelo de CRSC se observa que un 40\% lograron superar las 200 transacciones mensuales. Sólo un 20\% de las participantes permanecieron en el rango menor a 100. Entiéndase que al hablar de transacciones se refiere a los contactos con consumidores y/o clientes que concretaron una compra con el establecimiento pudiendo ser éstas de cualquier monto y por cualquier vía.

Orientando. Temas de Asia Oriental. Sociedad, Cultura y Economía. Revista editada por el Centro de Estudios China-Veracruz de la Universidad Veracruzana, México (Centro de Estudios APEC) / año 10 / número 21/ 


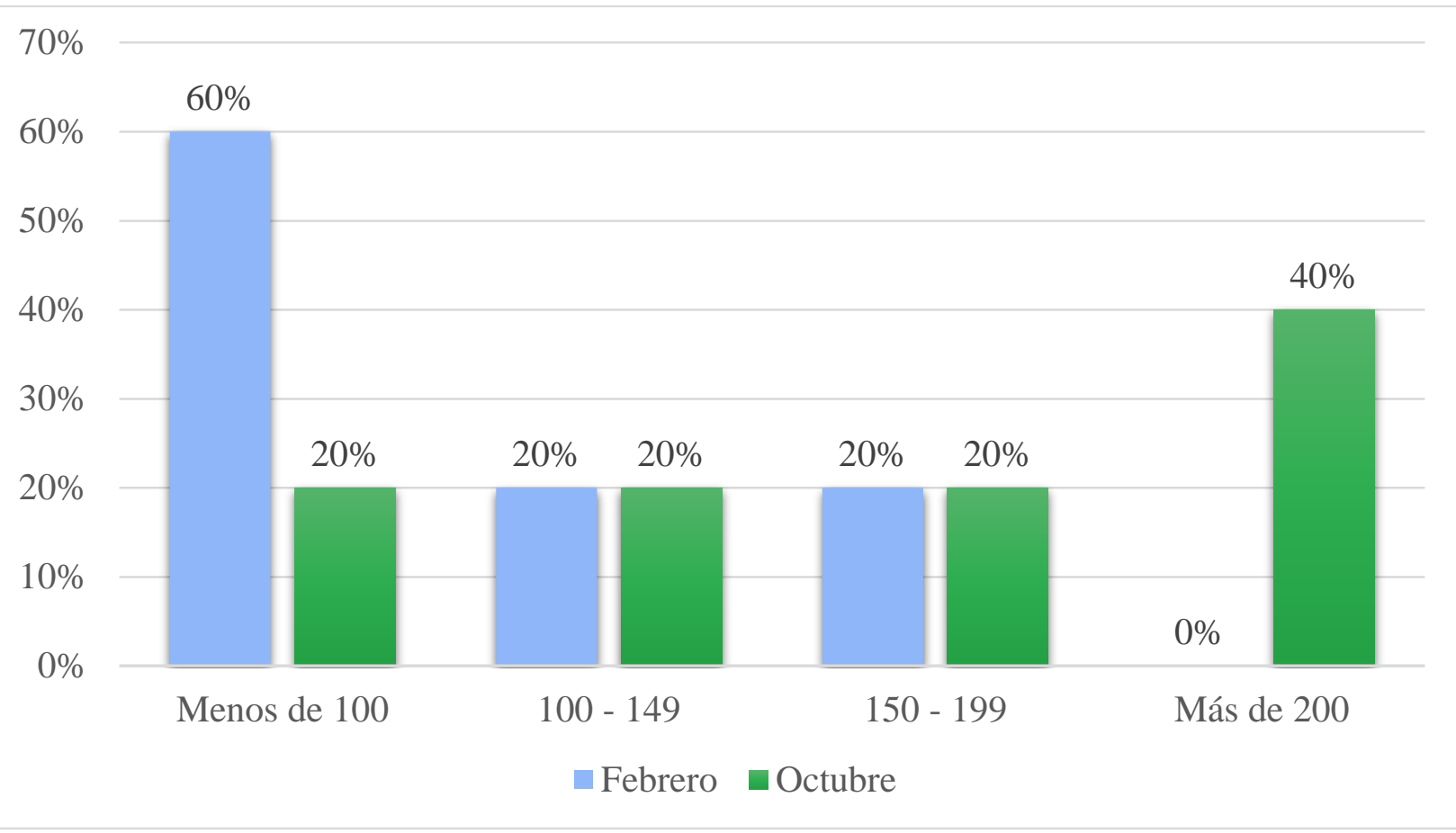

Figura 1. Comparativa del volumen de transacciones febrero/octubre 2020

Fuente: Elaboración propia.

El número de transacciones es un dato importante porque otorga una idea del flujo de operaciones con clientes, lo cual por supuesto podría variar dependiendo de la naturaleza de los bienes y servicios que la cooperativa ofrezca. Sin embargo, el valor en dinero de esas transacciones representa la parte determinante del análisis. La aplicación del modelo generó un importante incremento del rango de ingresos: en febrero el 60\% de las cooperativas generaban flujos mensuales entre los $\$ 10,000$ pesos mexicanos y los $\$ 17,999$ pesos mexicanos, y tan sólo un $20 \%$ de ellas registraban flujos que superaban los \$25, 000 pesos mexicanos. Durante el mes de octubre del 2020, el 80\% de las cooperativas participantes afirmaron que sus ingresos superaron los $\$ 25,000$ pesos mexicanos durante ese periodo (Figura 2). Ante este fenómeno, cabe resaltar que el 20\% de las cooperativas cambiaron de actividades con la intención de atender necesidades emergentes ante la pandemia generada

Orientando. Temas de Asia Oriental. Sociedad, Cultura y Economía. Revista editada por el Centro de Estudios China-Veracruz de la Universidad Veracruzana, México (Centro de Estudios APEC) / año 10 / número 21/ 
por la Covid-19, lo que propició que desarrollaran algunos productos incluso fuera de las actividades a las que acostumbraban realizar, manteniendo su fuente de ingreso que se registró durante el mes de octubre del 2020.

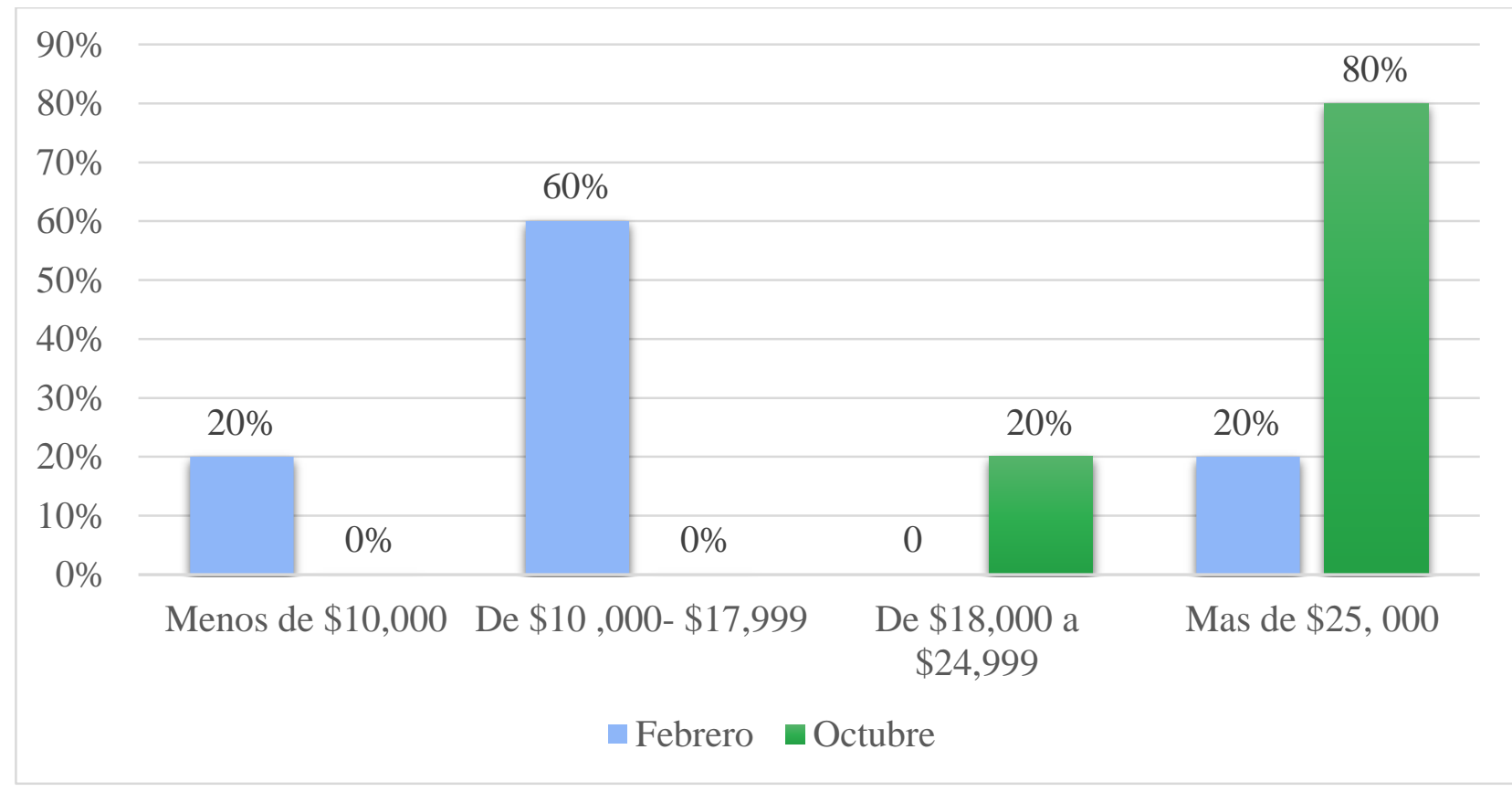

Figura 2. Comparativa de ingresos mensuales en pesos mexicanos febrero/octubre del 2020

Fuente: Elaboración propia.

Se considera que el incremento en los rangos de ingreso de las cooperativas es un hecho muy relevante, porque como se observa en la Figura 2 un $40 \%$ de las cooperativas lograron ascender al siguiente de rango de ingreso, y de ese $20 \%$ de cooperativas que facturaban menos de \$10, 000 pesos mexicanos en febrero, lograron saltar a otros rangos de ingreso, toda vez que en octubre de 2020, todas las cooperativas estaban por encima del nivel menor y un $80 \%$ en el nivel de ingreso mayor. Asimismo, incrementó la percepción que tienen los socios respecto a la influencia que atribuyen a los medios digitales a sus ingresos, en febrero de 2020 la mayoría de las cooperativas consideraban que los medios digitales influían apenas entre un $20 \%$ y $49 \%$ en sus ingresos. Para octubre del mismo año, un $40 \%$ de las cooperativas Orientando. Temas de Asia Oriental. Sociedad, Cultura y Economía. Revista editada por el Centro de Estudios 
consideran que el uso de medios digitales impactó en un rango de $80 \%$ y $100 \%$ en sus ingresos mensuales.

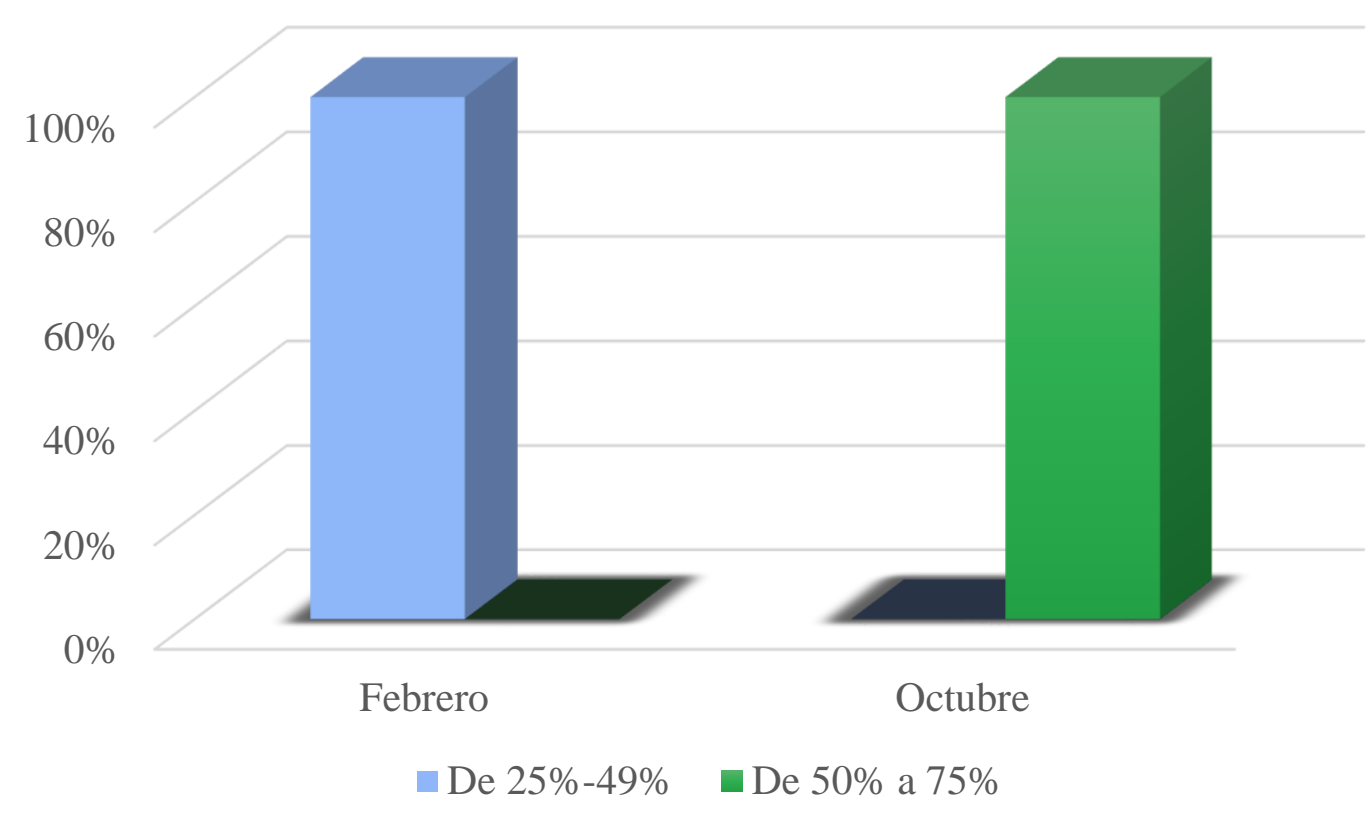

Figura 3. Comparativa del porcentaje de excedente respecto a los ingresos febrero/octubre 2020

Fuente: Elaboración propia.

Los excedentes (llamados también remanentes) en una cooperativa corresponden a las ganancias económicas que obtiene después de solventar los pasivos, fondos sociales e impuestos correspondientes. Lo que en una sociedad de capitales convencional como en una Sociedad Anónima le llamaríamos “utilidad”. En un ejercicio aplicado durante un mes, es complejo calcular el nivel de excedente considerando que contablemente es un cálculo que se realiza al cierre del ejercicio anual. No obstante, las cooperativas suelen efectuar procedimientos de "anticipos a excedentes", que son una especie de cortes periódicos, donde de ser necesario, distribuyen parte de esos beneficios o remanentes según la cooperativa lo determine.

Orientando. Temas de Asia Oriental. Sociedad, Cultura y Economía. Revista editada por el Centro de Estudios

China-Veracruz de la Universidad Veracruzana, México (Centro de Estudios APEC) / año 10 / número 21/ 
En la Figura 3 puede observarse que todas las cooperativas que participaron incrementaron los niveles de excedentes aproximados respecto a los ingresos de octubre y en comparación con febrero del 2020. Este dato, explica que las cooperativas lograron un mejor aprovechamiento de sus aportaciones sociales. Hace sentido completamente considerar que con los incrementos en las transacciones y en el nivel de ingresos, el aprovechamiento económico en los excedentes sea mayor pasando de un rango de $25 \%$ y $49 \%$ al de $50 \%$ y $75 \%$.

\section{Discusión}

La aplicación del modelo chino de CRSC acompañada de la estrategia O2O (online-offline) es un ejercicio interesante que brinda herramientas a las cooperativas, para que sus productos y servicios incrementen sus probabilidades de colocación en diferentes mercados. En forma similar, Yue (2016), determina que este modelo es una forma de generar desarrollo regional involucrando a la comunidad local, gracias al vasto potencial que tiene para integrar al comercio tradicional con una plataforma de bienes y servicios locales en línea.

Sin embargo, no se trata de un modelo que pueda — ni deba - replicarse exactamente igual sin considerar antes las características del contexto, necesidades de los actores y alcances esperados de quien pretenda tomarlo como referente, será fundamental considerar la viabilidad en tres aspectos: en primer lugar, lo social: toda cooperativa que se constituye plasma de inicio en sus objetivos sociales las líneas de acción que determinarán su existencia, pero debe tener en cuenta que existe un carácter económico en la cooperativa y que sólo la procuración, nunca por encima de las personas, será el medio de conquistas solidarias en la organización, mediante la educación cooperativa y la vinculación legislativa para un mayor entendimiento del sector (Guo et al., 2007).

En segundo lugar, lo técnico: pese a que las cooperativas en la ciudad de Xalapa no cuentan con todas las características de infraestructura tecnológica que tienen los ejemplos cooperativos en China, se puede poner en marcha la estrategia considerando los elementos Orientando. Temas de Asia Oriental. Sociedad, Cultura y Economía. Revista editada por el Centro de Estudios 
esenciales del modelo para beneficiarse de esquemas comerciales que impulsen a las empresas cooperativas, sin necesidad de realizar grandes inversiones y ciñéndose a los recursos disponibles de la sociedad; y en tercer lugar, lo institucional: si bien no existen las mismas condiciones en los gobiernos locales como las hubo en China, donde el gobierno dispuso de recursos para organizar a las familias en cooperativas (Poirier, 2011), tampoco es necesario que las cosas sucedan exactamente igual, actualmente se cuenta con el marco legal suficiente y la apertura en los gobiernos locales para desarrollar las actividades cooperativas bajo el esquema abordado en este estudio.

Considerando estos elementos y los resultados obtenidos se reconoce que las cooperativas xalapeñas pueden fortalecer sus actividades comerciales mediante la implementación de estrategias multicanales de e-commerce basadas en la experiencia china de cooperativas rurales de suministros y comercialización; porque no se trata sólo de vender por vender, sino de contribuir a mejorar la accesibilidad de los productos, los beneficios al consumidor y la experiencia como clientes que se traducirán en incrementos al intercambio de bienes y servicios cooperativos (Guo, 2017).

\section{Conclusión}

Los beneficios que se observaron durante el análisis de los resultados constituyen un esquema capaz de impulsar a las cooperativas locales y coadyuvar al desarrollo de estas organizaciones solidarias que sirven a la sociedad, ofreciendo bienes y servicios, atendiendo problemáticas y resolviéndolas para cubrir sus necesidades particulares y colectivas. El panorama es alentador porque la comercialización y distribución en las cooperativas encuentra una interesante propuesta de valor, basada en la experiencia china, que se resume en cinco pilares económicos que impulsan el desarrollo de las organizaciones cooperativas: 1) identidad cooperativa; 2) medios digitales; 3) más transacciones; 4) mayores ingresos, y 5) mejores excedentes, los cuales son completamente compatibles con la visión de empresa solidaria, constituyendo una forma para alcanzar los beneficios esperados en la comunidad de socios

Orientando. Temas de Asia Oriental. Sociedad, Cultura y Economía. Revista editada por el Centro de Estudios China-Veracruz de la Universidad Veracruzana, México (Centro de Estudios APEC) / año 10 / número 21/ 
cooperativistas, gracias a la integración del comercio tradicional con una plataforma de bienes y servicios locales en línea.

\section{Referencias}

Alianza Cooperativa Internacional (2018). Qué es una cooperativa. Bruselas, Bélgica. Recuperado de https://www.ica.coop/es/cooperativas/que-es-una-cooperativa

Arango, J. M. (2005). Manual de cooperativismo y economía solidaria. Recuperado de https://ebookcentral.proquest.com

Ávila, A.J. y Pérez, A. (2019). Expresiones de la Economía Social en el comportamiento de las microempresas familiares. Revista Otra Economía, vol. 12, n. 21:54-68, enerojunio 2019. ISSN 1851-4715

Coraggio, J. L. (2002). La economía social como vía para otro desarrollo social. Recuperado de

http://www.top.org.ar/ecgp/FullText/000000/CORAGGIO\%20Jose\%20Luis\%20-

$\% 201 \mathrm{\%} \% 20$ economia\%20social.pdf

Fritz-Krockow, B. (1986). Evaluación del cooperativismo mexicano. Comercio Exterior, 36 (9), 789- 796

Galindo, M. M. Á., \& Carrasco, M. I. (2009). Diccionario de economía social. Recuperado de https://ebookcentral.proquest.com

Gobierno del Estado de Veracruz (2018). Gaceta Oficial del Órgano del Gobierno del Estado de Veracruz de Ignacio de la Llave. Tomo CXCVII, núm. ext. (252). Recuperado de https://sisdti.segobver.gob.mx/siga/doc gaceta.php?id=1524

Galindo, M. M., \& Carrasco, M. I. (2009). Diccionario de economía social. Recuperado de https://ebookcentral.proquest.com

García, L.P y Vázquez, P. (2013). Beneficios Fiscales de las Sociedades Cooperativas de Producción y de Servicios. IPN. Recuperado de https://tesis.ipn.mx/handle/123456789/13418

Guo, G. (2017). Study on O2O Mode Innovation of Organic Vegetables in Hubei Province. International Symposium 2017--Asia-Pacific Public Administration, pp 28-33. ISBN: 978-1-946788-05-4.

Izquierdo, M. E. (2019). Problemas en las cooperativas mexicanas que atentan contra el principio de autonomía e independencia. Boletín de la Asociación Internacional de Derecho Cooperativo, 2019 (55), 35-54, doi: http://dx.doi.org/10.18543/baidc-552019pp35-54

Laville, J. y García, J. (2009). Crisis capitalista y economía solidaria. Una economía que emerge como alternativa real, Icaria, Barcelona.

Martínez, A. (2015). Las cooperativas y su acción sobre la sociedad. REVESCO. Revista de Estudios Cooperativos, núm. 117, enero-abril, pp. 34-49, doi: http://dx.doi.org/10.5209/ rev_REVE.2015.v117.48144

Orientando. Temas de Asia Oriental. Sociedad, Cultura y Economía. Revista editada por el Centro de Estudios 
Martínez, M. (2013). La economía social y solidaria (una mirada desde la teoría y la práctica). Cuadernos de investigación, Universidad Ibero Puebla.

Olmedo, R. (2017). El cooperativismo en México. Una alternativa en análisis. Iberoamérica Social. Recuperado de https://iberoamericasocial.com/cooperativismo-mexico-unaalternativa-analisis/

Oulhaj, L. (2013). Breve revisión conceptual del Tercer Sector. Miradas sobre la economía social y solidaria en México, UIA - Puebla, México, p.23

Pasquier, M. (2015). Innovation is everywhere: O2O: Why China leads the "Online to offline" revolution. Singapur: Innovation is Everywhere. Recuperado de https://www.innovationis everywhere.com /o2o-why-china-leads-the-online-tooffline-revolution/

Pérez, J.C., Etxezarreta, E. y Guridi, L. (2008). ¿De qué hablamos cuando hablamos de Economía Social y Solidaria? Concepto y nociones afines. XI Jornadas de Economía Crítica, Bilbao Ecocri 2008. Recuperado de: https://www.economiasolidaria.org/sites/default/files/ Economia_social_y_solidaria_concepto_nociones.pdf

Poirier, Y. (2011). Economía Social y Solidaria en la zona rural de China. Boletín Internacional de Desarrollo Local Sostenible \#81. Recuperado de: http://www.socioeco.org/bdf_fiche-document-1716_es.html

Rojas Coria, R. (1984): Tratado de Cooperativismo mexicano. Fondo de Cultura Económica. México D.F.

Rojas Herrera, J. J. (2014). La formación del movimiento cooperativo en México:

antecedentes organizacionales y momento constitutivo. México: UACh-Juan Pablos Editor

Xiangyu G., Henehan, B, \& Schmit, T. (2008). Rural Supply and Marketing Cooperatives in China: Historical Development, Problems and Reform. Recuperado de: https://www.researchgate.net/publication/238084223_Rural_Supply_and_Marketin g_Cooperatives_in_China_Historical_Development_Problems_and_Reform

Xinhuanet (2018). Xinhua News Agency: China impulsa reforma de cooperativas rurales de suministro y comercialización. Beijing, China: Xinhua. Recuperado de http://spanish.xinhuanet.com/2018-01/13/c_136891872.htm

Xinhuanet (2019). Xinhua News Agency: China establece más de 50.000 cooperativas agrícolas en primera mitad de 2019 para reducir la pobreza. Beijing, China: Xinhua. Recuperado de http://spanish.xinhuanet.com/2019-08/01/c_138275183.htm

Yue, G. (2016). The Study of the Application of O2O E-Commerce Model in China. WHICEB 2016 Proceedings 51. Recuperado de http://aisel.aisnet.org/whiceb2016/51

Orientando. Temas de Asia Oriental. Sociedad, Cultura y Economía. Revista editada por el Centro de Estudios 